\title{
Rastreio do cancro prostático: o actual paradigma da medicina centrada na pessoa
}

José Agostinho Santos*

\section{RESUMO}

Desde há alguns anos que o rastreio do cancro prostático através do Antigénio Específico da Próstata (PSA) tem emergido, entre a literatura médica, pela discussão em torno da sua razão efectividade/risco. A U.S. Preventive Services Task Force (USPSTF) fez, em 2012, uma recomendação contra o rastreio do cancro da próstata, concluindo que existe evidência considerável de que este não tem um saldo positivo quanto a vantagens/desvantagens. O impacto das recomendações da USPSTF poderá notar-se na maior atenção disponibilizada pelo médico na actualização quanto às normas produzidas pelas outras sociedades científicas. Parece ser muito relevante que o médico conheça no que a evidência científica se traduz.

Os resultados dos ensaios clínicos Prostate, Lung, Colorectal and Ovarian Cancer Screening Trial (PLCO) e European Randomized Study of Screening for Prostate Cancer (ERSPC) constituem a principal evidência, embora ambos apresentem limitações a apontar. De uma análise global destes estudos, sobressai que o número de homens que evitaram a morte por cancro prostático por terem sido submetidos ao rastreio foi reduzido. O primeiro estudo não mostrou qualquer redução da mortalidade $[R R=1,09(0,87-1,36)]$, enquanto o segundo mostrou que o número de mortes por cancro prostático foi de 5 para 1000 homens no grupo sem rastreio e de 4 para 1000 homens nos submetidos a rastreio $[R R=0,79(0,68-0,91)]$, após 11 anos de seguimento. Há evidência de que 100 a 200 em 1000 homens rastreados terão um falso positivo, a maioria dos quais será biopsada. Entretanto, uma grande parte dos doentes com carcinomas prostáticos detectados por rastreio poderá ser submetida a cirurgia, radioterapia ou terapêutica de privação androgénica.

Este artigo pretende salientar os aspectos que não recomendam a solicitação sistemática do PSA e favorecem uma abordagem holística que envolva a pessoa na ponderação das questões relativas ao rastreio e do saldo vantagens/desvantagens.

Palavras chave: Detecção Precoce de Cancro; Rastreio Universal; Neoplasia Prostática.

\section{AS NORMAS E AS SUAS LEITURAS}

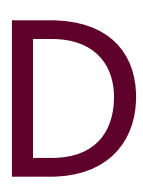
esde há alguns anos que o rastreio do cancro prostático tem emergido, entre a literatura médica, pela discussão em torno da sua razão efectividade/risco. ${ }^{1}$ O Antigénio Específico da Próstata (PSA) começou a ser usado como método de rastreio nos anos 90. Porém, desde que foram publicados na primeira década do século XXI os resultados de ensaios clínicos aleatorizados e controlados (cuja qualidade era superior à dos estudos observacionais), sabe-se que o rastreio deste carcinoma através do PSA não tem o benefício que era anteriormente considerado. ${ }^{1-5}$ Esses mesmos ensaios clínicos, que merecerão maior detalhe abaixo, produziram novos resulta-

*Assistente de Medicina Geral e Familiar, USF Dunas, ULS - Matosinhos dos após 11 anos de seguimento, cuja publicação ocorreu já na segunda década do século XXI, trazendo uma maior consistência e solidez às recomendações elaboradas pelas sociedades científicas. ${ }^{6-7}$

Uma dessas sociedades foi a U.S. Preventive Services Task Force (USPSTF) que, em 2012, fez uma recomendação contra o rastreio do cancro da próstata, concluindo que existe certeza moderada-elevada de que o rastreio através do PSA não tem saldo benéfico ou que os riscos ultrapassam os benefícios. Esta recomendação sobressaiu pelo contraste estabelecido com as suas normas de 2008 (em que não fazia uma recomendação nem a favor nem contra o rastreio do cancro prostático), agitou a comunidade científica e veio motivar uma corrente de outras publicações. . $^{4-5,8-9}$

A European Association of Urologysugere que, no caso 
de se iniciar o rastreio do cancro prostático através do PSA sérico, uma primeira colheita deverá ser realizada aos 40 anos, enquanto a American Urological Association propõe actualmente um início aos 55 anos. ${ }^{3,10} \mathrm{~A}$ Associação Europeia avança que, se o PSA for $\leq 1 \mathrm{ng} / \mathrm{mL}$, o risco de desenvolver cancro prostático é baixo, pelo que uma nova medição poderá ser novamente solicitada após 8 anos. ${ }^{10}$ Todas as recomendações actuais para este rastreio integram o PSA sérico, sendo o toque rectal e a ecografia prostática incluídas em algumas normas, ${ }^{3,10}$ embora nenhuma destas duas técnicas tenham benefício comprovado quanto a resultados orientados para o paciente. ${ }^{1,3}$ A Direcção-Geral da Saúde (DGS) redige, nas linhas da Norma de Orientação Clínica (NOC) emitida em 2012 e actualizada em 2014, que o PSA sérico não deverá ser solicitado no rastreio populacional de cancro prostático, fundamentando que «a utilização do PSA no rastreio em indivíduos assintomáticos é controversa e aguarda finalização de estudos prospectivos randomisados que evidenciem, inequivocamente, o balanço entre os beneficios de um diagnóstico precoce e os efeitos nefastos de falsos positivos». Escreve, contudo, que «a detecção precoce (rastreio oportunístico) só deve ser facultada a homens que o solicitem, depois de devidamente informados sobre os beneficios e os riscos de sobrediagnóstico e hipertratamento, através da determinação do PSA total, com ou sem palpação da próstata». ${ }^{11}$

Nenhuma das sociedades científicas referidas acima (assim como a American Cancer Society, American Society of Clinical Oncology, , American College of Physicians, American College of Preventive Medicine, entre outras) recomenda, na versão mais recente das suas normas, o rastreio sistemático e universal e sem considerar as suposições e dúvidas em seu torno. Não recomendam, portanto, o início e desencadeamento do rastreio em que a atitude clínica proactiva seria a primeira força-motriz mas, pelo contrário, alertam os clínicos para a necessidade de explanação dos prós e contras actuais, junto do seu paciente. ${ }^{1,3,10}$ Este processo de esclarecimento seria a base para uma decisão partilhada com o homem, algo que, na opinião do autor, apela certamente a competências próprias do médico de família, como os cuidados centrados na pessoa e a gestão dos cuidados de saúde primários, constituindo, por si só, um paradigma da medicina centrada na pessoa. ${ }^{12-13}$ Este aconselhamento clínico desvenda, desde já, um possível maior tempo de consulta, pela dispensa temporal na exploração destas diversas peculiaridades relativas ao conhecimento actual sobre o rastreio do cancro prostático através do PSA, usando uma linguagem que se adapte ao meio psicossocial único de cada homem. ${ }^{13-17}$

O contexto da incerteza médica, que oscila entre «os possíveis benefícios» (enraizados desde a introdução do PSA nos anos 90) e os «eventuais riscos», parece aliar-se a uma forte dependência sociocultural deste rastreio desde que foi instituído, trazida pelo paciente para dentro do gabinete médico. ${ }^{13-15}$ É possível, então, que subsista alguma dúvida se tais normas serão suficientes para abrandar uma atitude mais proactiva do médico na solicitação do PSA, atitude essa encorajada pelo desejo (ou consentimento desinformado?) do seu paciente e pela pressão exterior e exigente quanto aos limites do tempo de consulta. ${ }^{16-18}$ Não se questiona, portanto, se os clínicos conhecem as normas desde a sua publicação, realçando que a relação entre a prática particular do clínico e as NOC produzidas por entidades científicas envolve um processo complexo que vai desde o conhecimento à adesão. ${ }^{19}$

O impacto das recomendações da USPSTF poderá notar-se, paradoxalmente, na maior atenção disponibilizada pelo médico na actualização quanto às NOC produzidas por outras sociedades científicas. ${ }^{16-17,20}$ Segundo a opinião do autor deste artigo, o carácter contra-recomendativo da publicação da USPSTF quanto ao rastreio do cancro prostático poderá incitar fortemente o clínico para uma comparação com o que outras sociedades de renome redigem nas linhas das suas normas e para a procura de esclarecimento. Mesmo que o clínico considere que a evidência não é suficiente para recomendar contra o rastreio, ele irá tendencialmente ao encontro da recomendação de uma decisão caso a caso e de acordo com o desejo do paciente informado, onde uma atitude clínica proactiva não é jamais recomendada. ${ }^{16-18}$

Sabe-se, hoje, que o risco de diagnóstico de carcinoma prostático ronda os $15,9 \%$ mas que o risco de morte por esta neoplasia é cerca de $3 \%{ }^{4,11,21}$ Parece ser, portanto, muito relevante que o clínico, em particular o médico de família, conheça no que essa evidência se traduz.

\section{RASTREIO ATRAVÉS DO PSA: A EVIDÊNCIA QUE CONVIDA À REFLEXÃO}

O valor sérico do PSA é portador de um potencial para falsos positivos, uma vez que poderá estar au- 
mentado (globalmente $\geq 4,0 \mathrm{ng} / \mathrm{mL}$, embora a American Urological Association estabeleça diferentes cut-offs de acordo com a idade e a raça) em diversas condições clínicas benignas como hiperplasia benigna da próstata, prostatite, biópsia prostática, ejaculação, treino de ciclismo ou exercício físico vigoroso. ${ }^{1-3,10}$

Por outro lado, o cancro prostático constitui uma patologia muito frequente mas igualmente oculta, em que a maioria dos casos nunca se apresentará sob qualquer quadro clínico na vida do homem. De facto, estudos de autópsias revelaram que aproximadamente um terço dos homens com idade compreendida entre os 40 e os 60 anos tem um cancro prostático (histologicamente evidente e clinicamente sem expressão), sendo que esse valor se eleva até aos três quartos nos homens com idades $\geq 75$ anos. Também revelaram que a maioria desses cancros consiste em lesões circunscritas e com boa diferenciação.-4,11,21-22

Sendo certo e cientificamente comprovado que o rastreio do cancro prostático através do PSA conduz à identificação de um grande número desses tumores assintomáticos, está também comprovado que uma grande percentagem desses carcinomas evolui a baixa velocidade, pelo que permanecerão assintomáticos ao longo da vida dos homens afectados que entretanto morrerão de outras causas que não por esta neoplasia. ${ }^{1-4,11,21}$ Este aspecto fez emergir o conceito de sobrediagnóstico, que consiste no evento de detecção clínica de patologias que sem investigação médica permaneceriam silenciosas e nunca derivariam num quadro sintomático ou em morte. No caso particular do cancro da próstata, é calculado que esse sobrediagnóstico se situe na ordem dos 17 a $50 \%$, isto é, 17 a 50 dos 100 cancros detectados devido ao rastreio nunca se tornariam sintomáticos. ${ }^{3-4,21}$ Para os outros homens a quem o rastreio detectou tumores que se tornariam futuramente sintomáticos nas suas vidas, os mesmos estudos revelam que a maioria (que são aqueles que apresentam níveis séricos de PSA $<10 \mathrm{ng} / \mathrm{mL}$ ) também não retira grande benefício: se esses tumores fossem deixados intactos até à sua detecção por início dos sintomas e não por rastreio o resultado para a duração das suas vidas seria semelhante. ${ }^{4,21}$ Trata-se de um dado revelado por investigação recente que comparou indivíduos submetidos a prostatectomia com os que mantêm apenas vigilância: após anos de seguimento, os índices de mortalidade são iguais (excepto no subgrupo dos homens prostatectomizados e com valores de PSA $>10 \mathrm{ng} / \mathrm{mL}$ no rastreio, em que há uma redução de $7 \%$ de mortalidade por carcinoma prostático comparativamente ao grupo sob vigilância). ${ }^{4}$

A assimilação do conceito de sobrediagnóstico parece, portanto, ser de grande importância numa abordagem holística de cada pessoa única, dotada dos seus valores individuais, das suas prioridades na vida e das suas expectativas. Este é, então, distante do significado de falso positivo, pois este implica a suspeita inicial de um diagnóstico que, posteriormente, não é confirmado. $^{3-4,21}$ Ambos os conceitos encerram, no entanto, as duas maiores questões que surgem no momento em que o médico e o seu paciente discutem um início do rastreio (abordagem pré-rastreio). Quando este é iniciado, os estudos mais recentes conferem-nos dados relevantes quanto aos seus benefícios e riscos.

Quanto aos benefícios do desencadeamento do rastreio do cancro prostático através do valor sérico do PSA, existe evidência de que o procedimento clínico conduz à identificação de uma percentagem considerável de carcinomas prostáticos assintomáticos. Os resultados que conferem maior consistência a esta evidência actual são os dos dois ensaios clínicos aleatorizados e controlados, o Prostate, Lung, Colorectal and Ovarian Cancer Screening Trial (PLCO), com inclusão de 76685 homens com idades entre 55 e 74 anos; PSA anual durante 6 anos e toque rectal anual durante 4 anos versus cuidados habituais no contexto da consulta generalista; desenvolvido nos EUA, onde o uso do PSA era já disseminado na prática clínica antes do início do estudo, pelo que a taxa de contaminação no grupo controlo com pelo menos um valor sérico de PSA atingiu $40 \%$ no primeiro ano de estudo e o European Randomized Study of Screening for Prostate Cancer (ERSPC), com 182160 homens incluídos com idades entre 50 e 74 anos; diferentes esquemas de rastreio de acordo com o país, desde PSA bianual até PSA quadrienal ou de 7 em 7 anos; desenvolvido em sete países europeus onde o uso do PSA não se encontrava tão disseminado, pelo que a taxa de contaminação entre os controlos foi estimada em $15 \% .^{1-4,8}$ Estes dois grandes ensaios forneceram dados que mereceram uma atenta interpretação (inclusivamente, em duas meta-análises publicadas em 2010 e 2011), ,23-24 sendo o mais significativo aquele revelador de que foi baixo o número de ho- 
mens que evitaram a morte por cancro prostático após terem sido submetidos ao rastreio. ${ }^{6-7} \mathrm{O}$ ensaio americano não mostrou redução da mortalidade $[R R=1,09$ $(0,87-1,36)],{ }^{4,6}$ enquanto que o europeu estabeleceu um ligeiro diferencial quanto ao número de mortes por cancro prostático após 11-13 anos de seguimento: 5 para 1000 homens no grupo sem rastreio e 4 para 1000 homens nos submetidos a rastreio $[R R=0,79(0,68-$ 0,91)]. ${ }^{4,7}$ Uma breve referência deverá ser feita ao Göteborg trial, de menor dimensão, com mais limitações metodológicas e em que $60 \%$ dos pacientes entraram no ERSPC: após 14 anos de seguimento obteve-se como resultado um $R R=0,56(0,39-0,82){ }^{25}$

A assimetria de resultados entre os dois estudos é justificada pelas peculiaridades de cada um deles, algumas delas constituindo limitações metodológicas: a referida taxa de contaminação no grupo controlo do PLCO poderá ter reduzido as diferenças com o grupo de intervenção em termos de resultados finais nesse ensaio; também o facto dos pacientes do grupo de intervenção do ERSPC diagnosticados com carcinoma prostático terem sido orientados e tratados em centros de referenciação terciária (ao contrário dos controlos com esse diagnóstico, que foram abordados nos cuidados de saúde hospitalares habituais) deverá ser considerado. ${ }^{1-4,8,13}$ Outros prováveis factores geradores dessa divergência são a menor periodicidade estabelecida para o rastreio e a maior percentagem de homens diagnosticados submetidos a prostatectomia radical no braço do grupo de intervenção no ensaio americano assim como o facto de, no ERSPC, o benefício para a mortalidade específica ter ocorrido de forma estatisticamente significativa graças aos resultados de centros que usaram limiares de PSA inferiores ou iguais a $3.0 \mu \mathrm{g} / \mathrm{L} .1,5,26$

Estes ensaios ainda decorrem actualmente, aguardando-se os dados correspondentes a um seguimento superior ao tempo de latência definido para este rastreio (15 anos) e que serão os mais definidores quanto ao valor do mesmo na redução da mortalidade específica por carcinoma prostático. ${ }^{1,27}$ Contudo, é nestes resultados de 11 anos de follow-up em países europeus que a USPSTF se baseia ao salientar que o benefício do rastreio através do PSA assenta na evicção de 1 morte por cancro prostático por cada 1000 homens rastreados e que, portanto, cerca de 1000 homens terão que ser convidados a rastreio e terá que ser feito o diagnóstico de carcinoma prostático a 37 homens para que seja evitada 1 morte por esta neoplasia. ${ }^{4}$

Uma sub-análise do ERSPC recentemente publicada demonstrou, entretanto, uma diminuição de $30 \%$ no risco de desenvolver metástases no grupo da intervenção. Este dado poderá assumir particular relevância na interpretação dos resultados globais deste ensaio clínico aos 11 anos de seguimento, especulando-se que, com o decorrer do estudo, se acentuem as diferenças na mortalidade específica da doença e na qualidade de vida dos homens entre os dois grupos. Os próximos anos revelarão dados que permitirão análises objectivas e não meramente especulativas. ${ }^{27}$

Relativamente aos riscos inerentes a este rastreio, foi já evidenciado potencial para falsos positivos retido na medição do PSA, que resultam em dano psicológico para os homens que iniciam neste ponto uma elevação do seu estado basal de ansiedade dominada pela possível confirmação de um tumor maligno. ${ }^{3-4,21}$ Estes homens são submetidos a um maior número de exames complementares do que os que não obtêm falsos positivos. A literatura indica que cerca de 15 a $20 \%$ dos homens rastreados ao longo de 10 anos terão um valor que resultará em biópsia para um diagnóstico diferencial, dependente do valor-limite do PSA usado ou do intervalo entre medições. ${ }^{4}$ Por sua vez, $33 \%$ dos pacientes biopsados vivenciam dor, febre, hemorragia (hematúria, hematospermia) ou dificuldade urinária e 0,5-1\% necessitam de hospitalização pós-biópsia. ${ }^{3-4}$

De uma análise global, a USPSTF realça que existe evidência de que 100 a 200 em 1000 homens rastreados terão um valor falso positivo do PSA e que a maioria destes será biopsada, algo que traz consequentes lesões psicológicas e orgânicas em parte deles. ${ }^{4}$ Esta sociedade também evidencia que $90 \%$ dos homens americanos com tumores detectados por rastreio serão submetidos a cirurgia, radioterapia ou terapêutica de privação androgénica. ${ }^{3-4,8,21}$ Estas são opções terapêuticas que apresentam, por sua vez, um elevado custo para parte dos homens tratados: 10 a 70 dos 1000 submetidos a prostatectomia terão complicações graves. Estima-se que 200 a 300 dos 1000 homens tratados com cirurgia ou radioterapia sofrerão de incontinência urinária e disfunção eréctil. Já a terapêutica de privação androgénica resulta em disfunção eréctil em 400 dos 1000 homens tratados. A USPSTF calcula que cerca de 29 em cada 1000 homens rastreados 
terão disfunção eréctil e 18 terão incontinência urinária depois do tratamento para cancro prostático. ${ }^{4,28}$

Assim, a partir de um balanço crítico entre as vantagens e desvantagens, esta mesma sociedade científica tornou pública, em Julho de 2012, a recomendação contra o rastreio do cancro da próstata através do PSA, considerando que «os benefícios em termos de mortalidade são reduzidos a potencialmente nenhuns e que os riscos são moderados a substanciais», salientando que não existe igualmente evidência que suporte um rastreio específico e dirigido aos homens dos 55 aos 69 anos de idade com risco aumentado (raça negra, história familiar de carcinoma prostático) nem um rastreio segundo um método alternativo ao PSA. ${ }^{3-4,11}$

Este saldo final negativo poderia colidir com os resultados de alguns estudos observacionais que têm documentado que a taxa de mortalidade por cancro prostático nos EUA tem vindo a decrescer lentamente desde os anos 90, altura da introdução do PSA sérico como método de rastreio. ${ }^{1,4,8,21}$ A taxa de mortalidade específica tem mostrado uma inversão também em Portugal, com uma diminuição de $3,1 \%$ por ano entre 1998 e $2006 .{ }^{28}$ Os autores da USPSTF salientam, porém, que essa redução da mortalidade no continente americano não poderá dever-se ao método de rastreio, pois a emergência do benefício com a introdução deste método apenas se evidenciaria após 10 a 15 anos da seu uso. Trata-se de um aspecto que tem suporte nos resultados do ERSPC cujo benefício notado na mortalidade apenas se verificou após 8 anos de seguimento. ${ }^{4,7-8,28-29}$ Bastos e colegas chegaram a conclusões semelhantes quanto ao declínio da taxa em Portugal, que se verificou concomitantemente com a introdução do PSA no final do século XX. Fica a dúvida, então, se esta redução não poderá estar relacionada em parte com o tratamento (possível factor de confundimento), que, embora possa condicionar redução de mortalidade, não objectiva necessariamente um aumento da qualidade de vida para aquele paciente. ${ }^{1,4,21}$

A American Urological Association recomenda, portanto, que este rastreio seja considerado em homens com 55 a 69 anos, com mais de 10 anos de esperança de vida e que, após terem sido devidamente informados dos riscos e benefícios conhecidos actualmente, queiram submeter-se ao rastreio. ${ }^{3}$ Algo semelhante recomenda a European Association of Urology, ${ }^{10}$ embora para idade de início inferior (40 anos), enquanto a Ame- rican Cancer Society sobe a idade de início para 50 anos, exceptuando os 45 anos para indivíduos de raça negra e antecedentes familiares de neoplasia maligna prostática. $^{30}$

Algumas formas que se pensam ser favorecedoras de redução dos danos para o paciente incluem aumento do cut-off do PSA para decisão para biópsia, integração dos valores séricos do PSA livre, da densidade do PSA ou da velocidade do mesmo. Estas formas carecem também de estudos que provem que, de facto, aumentam o benefício/risco ao reduzir o denominador. ${ }^{3-4}$ A NOC da DGS destaca que a relação PSA livre/PSA total é usada para apoio à decisão de biópsia quando o valor do PSA total se situa entre $4-10$ ng/mL e com palpação negativa. ${ }^{11}$

\section{CONCLUSÕES}

O rastreio sistemático do cancro prostático a homens em idades-alvo através do PSA sérico não dispõe de evidência científica robusta que suporte uma recomendação a seu favor. O objectivo primário do método de rastreio baseado no PSA é identificar e tratar homens cuja detecção e terapêutica reduzirão a sua morbilidade e mortalidade. Estudos revelam que o número de homens que experimentam este benefício é muito diminuto, alertando para o facto de que o rastreio poderá trazer mais prejuízo (morbilidade) do que benefício (redução de morbilidade e mortalidade). Nenhum dos ensaios clínicos em torno do rastreio ou do tratamento dos carcinomas prostáticos detectados a partir de um rastreio positivo demonstrou uma redução da taxa de mortalidade por todas as causas após 11 anos de seguimento. O ensaio clínico europeu ERSPC mostrou uma redução da mortalidade específica por cancro prostático, aguardando-se a publicação de novos dados após um maior tempo de seguimento que cubra com segurança o tempo de latência estimado.

A ausência de um benefício que ultrapasse os riscos com o rastreio do cancro da próstata através do PSA foi, assim, assumida pela USPSTF, lançando uma «pedra no charco». Tal efeito não se deve ao facto de ter conduzido a alterações imediatas nas atitudes clínicas da maioria dos médicos, mas sim ao facto de relembrar, de forma talvez mais acutilante, que a solicitação do PSA sérico carrega o potencial para iniciar uma cadeia diagnóstica que poderá ser danosa para o paciente. Carlsson e colegas estabelecem críticas às análises estatísti- 
cas e discussão da USPSTF, porém debruçam-se sobre este aspecto, escrevendo que é razoável recomendar contra a prática clínica actual, que constitui rastreio baseado no PSA e tratamentos subsequentes aos diagnósticos nos EUA, em que um terço dos homens com idade superior a 70 anos e $15 \%$ dos homens idosos com outras neoplasias (e, portanto, com esperança de vida reduzida) são submetidos ao rastreio com PSA. ${ }^{8}$

Desta análise geral de todas as premissas e evidências em torno do rastreio, o Médico de Família poderá resgatar os aspectos que não recomendam a solicitação do PSA de forma sistemática mas que favorecem uma abordagem holística que envolva a pessoa na ponderação das questões relativas ao rastreio e do saldo vantagens/desvantagens. Essa abordagem englobará a consideração de uma possível redução da taxa de mortalidade específica e de um aumento da taxa de danos, abrindo campo para uma decisão partilhada entre o médico e o utente. Tal apela a uma medicina centrada na pessoa (particularmente, nas suas preferências, expectativas e dúvidas), algo que exigirá um maior tempo dispendido nesta decisão partilhada. Todavia, poder-se-á pensar que os custos associados a este tempo alargado de consulta poderão ser largamente compensados pela poupança conseguida na prevenção quaternária em que tal poderá derivar. Definida como um conjunto de actividades que visam a evicção de procedimentos médicos excessivos, a prevenção quaternária passível de ser concretizada a este nível faz emergir a seguinte questão: será este rastreio um procedimento médico excessivo? Na opinião do autor deste artigo, o potencial para uma prevenção quaternária está evidente na neutralização parcial das solicitações do PSA sem discussão prévia com o utente e a atribuição do adjectivo «excessivo» a este rastreio variará de acordo com as características biológicas e psicossociais do homem que se senta no gabinete médico. Trata-se de factores inerentes à pessoa a ter em conta antes dessa atribuição, que não correrão o risco de ser esquecidos caso haja uma medicina centrada na pessoa única que cada homem representa.

Assim, sem benefício comprovado, a integração no rastreio do cancro prostático eleva a necessidade de uma reflexão conjunta no seio da relação médico-pessoa. A relevância deste ponto assenta na plataforma que ambos constroem no sentido de criar uma decisão partilhada, algo que só cimentará a relação e que relembra os números mais relevantes: a) benefício: 1 morte por cancro da próstata evitada por cada 1000 homens rastreados durante uma década; b) falsos positivos: por cada 1000 homens rastreados, 100 a 200 terão falsos positivos; c) dano: por cada 1000 homens rastreados, 29 terão disfunção eréctil e 18 terão incontinência urinária depois do tratamento para o carcinoma. Não deixa, contudo, de ser curioso que Richard Albin, o investigador que primeiro descobriu o PSA, tenha escrito numa comunicação em 2010 ao jornal New York Times que acredita actualmente que o uso desta molécula para rastreio do cancro prostático constitua um «desastre em saúde pública». ${ }^{31} \mathrm{~A}$ controvérsia continua.

\section{REFERÊNCIAS BIBLIOGRÁFICAS}

1. Croswell JM, Kramer BS, Crawford D. Screening for prostate cancer with PSA testing: current status and future directions. Oncology (Willinston Park). 2011;25(6):452-60, 463.

2. Brett T. Prostate specific antigen. Aust Fam Physician. 2011;40(7):497500.

3. Carter HB, Albertsen PC, Barry MJ, Etzioni R, Freedland SJ, Greene KJ, et al. Early Detection of Prostate Cancer: AUA Guideline. J Urol. 2013;190(2):419-26.

4. Moyer VA. Screening for prostate cancer: U.S. Preventive Services Task Force recommendation statement. Ann Intern Med. 2012;157(2):120-34.

5. U.S. Preventive Services Task Force. Screening for prostate cancer: U.S. Preventive Services Task Force Recommendation statement. Ann Intern Med. 2008;149(3):185-91.

6. Andriole GL, Crawford ED, Grubb RL 3rd, Buys SS, Chia D, Church TR, et al. Prostate cancer screening in the randomized Prostate, Lung, Colorectal, and Ovarian Cancer Screening Trial: mortality results after 13 years of follow-up. J Natl Cancer Inst. 2012;104(2):125-32.

7. Schröder FH, Hugosson J, Roobol MJ, Tammela TL, Ciatto S, Nelen V, et al. Prostate-cancer mortality at 11 years of follow-up. N Eng J Med. 2012;366(11):981-90.

8. Carlsson S, Vickers AJ, Roobol M, Eastham J, Scardino P, Lilja H, et al. Prostate cancer screening: facts, statistics and interpretation in response to the US Preventive Services Task Force Review. J Clin Oncol. 2012;30(21):2581-4.

9. Bull M, Schröder FH. Screening for prostate cancer - the controversy continues, but can it be resolved? Acta Oncol. 2011;50(Suppl 1):4-11.

10. Heidenreich A, Bastian PJ, Bellmunt J, Bolla M, Joniau S, Mason MD, et al. Guidelines on Prostate Cancer. Arnhem: European Association of Urology; 2010.

11. Direcção-Geral da Saúde. Prescrição e determinação do antigénio específico da próstata - PSA. Lisboa: DGS; 2014.

12. Wonca Europa. A definição europeia de Medicina Geral de Familiar. Wonca Europa; 2002. p. 6-14.

13. BarrattAL, Stockler MR. Screening for prostate cancer: explaining new trial results and their implications to patients. Med J Aust. 2009;191(4):226-9.

14. Kim SP, Karnes RJ. An Editorial Response to the USPSTF Prostate can- 
cer screening: the case for patient-centered shared decision-making. J Mens Health. 2012;9(1):5-8.

15. Sheridan SL, Harris RP, Woolf SH. Shared Decision Making About Screening and Chemoprevention: a suggested approach from the U.S. Preventive Services Task Force. Am J Prev Med. 2004;26(1):56-66.

16. Pollack CE, Noronha G, Green GE, Bhavsar NA, Carter HB. Primary care providers' response to the US Preventive Services Task Force draft recommendations on screening for prostate cancer. Arch Intern Med. 2012;172(8):668-70.

17. Walsh PC. Re: Primary Care Providers' Response to the US Preventive Services Task Force Draft Recommendations on Screening for Prostate Cancer. J Urol. 2012;188(4):1182.

18. Prasad SM, Drazer MW, Huo D, Hu JC, Eggener SE. 2008 US Preventive Services Task Force recommendations and prostate cancer screening rates. JAMA. 2012;307(16):1692-4.

19. Pathman DE, Konrad TR, Freed GL, Freeman VA, Koch GG. The awarenessto-adherence model of the steps to clinical guideline compliance. The case of pediatric vaccine recommendations. Med Care. 1996;34(9):873-89.

20. American Urological Association. AUA responds to new recommendations on prostate cancer screening [press release]. Linthicum, MD:AUA; 2011.

21. Welch HG, Black WC. Overdiagnosis in cancer. J Natl Cancer Inst. 2010;102(9):605-13.

22. Silva FC. Rastreio do cancro da próstata. Acta Urol. 2005;22(3):11-3.

23. Djulbegovic M, Beyth RJ, Neuberger MM, Stoffs TL,Vieweg J, Djulbegovic $B$, et al. Screening for prostate cancer: systematic review and meta-analysis of randomised controlled trials. BMJ. 2010;341:c4543.

24. Ilic D, O'Connor D, Green S, Wilt TJ. Screening for prostate cancer: an updated Cochrane systematic review. BJU Int. 2011;107(6):882-91.

25. Hugosson J, Carlsson S, Aus G, Bergdahl S, Khatami A, Lodding P, et al.
Mortality results from the Göteborg randomised population-basedprostate-cancer screening trial. Lancet Oncol. 2010;11(8):725-32.

26. Santos JA. Hiperplasia Prostática Benigna e PSA: o efeito dominó. Rev Bras Med Fam Comunidade. 2012;7(25):259-64.

27. Schröder FH, Hugosson J, Carlsson S, Tammela T, Määttänen L, Auvinen A, et al. Screening for prostate cancer decreases the risk of developing metastatic disease: findings from the European Randomized Study of Screening for Prostate Cancer (ERSPC). Eur Urol. 2012;62(5):745-52.

28. Bastos J, Botelho F, Pina F, Lunet N. Evolução da mortalidade por cancro da próstata em Portugal (1980 - 2006). Acta Med Port. 2011;24(4): 499-504.

29. Törnblom M, Eriksson H, Franzén S, Gustafsson O, Lilja H, Norming U, et al. Lead time associated with screening for prostate cancer. Int J Cancer. 2004;108(1):122-9.

30. Wolf AM, Wender RC, Etzioni RB, Thompson IM, D'Amico AV, Volk RJ, et al.American Cancer Society guideline for the early detection of prostate cancer: update 2010. CA Cancer J Clin. 2010;60(2):70-98.

31. Albin RJ. The great prostate mistake. New York Times, 2010.

\section{CONFLITOS DE INTERESSE}

O autor é editor da RPMGF.

\author{
ENDEREÇO PARA CORRESPONDÊNCIA \\ José Agostinho Santos \\ Rua Hernâni Torres, $73,4^{\circ}$ Dir \\ 4200 - 320 Porto \\ E-mail: zeagostinho@hotmail.com
}

Recebido em 31-07-2013

Aceite para publicação em 01-04-2014

\section{ABSTRACT}

\section{PROSTATE CANCER SCREENING IN THE LIGHT OF PERSON-CENTERED MEDICINE}

For several years, there has been debate regarding the cost effectiveness of prostate cancer screening. In 2012 the U.S. Preventive Services Task Force (USPSTF) recommended against screening for prostate cancer, concluding that there is considerable evidence that this is not beneficial. The impact of the recommendations of USPSTF may be noted in the guidelines produced by other scientific societies. Clinicians need to know the evidence.

The results of two randomized controlled trials, the Prostate, Lung, Colorectal and Ovarian Cancer Screening Trial (PLCO) and the European Randomized Study of Screening for Prostate Cancer (ERSPC), form the largest body of current evidence, although both have limitations. An analysis of the results of these trials shows a small reduction in prostate cancer deaths among men who were screened. The first trial showed no reduction in mortality $[R R=1.09$ ( 0.87 to 1.36$)$ ], while the latter trial showed that the number of deaths from prostate cancer was 5 per 1000 men in the group without screening and 4 per 1000 men undergoing screening [RR $=0.79(0.68$ to 0.91$)]$ after 11 years of follow up. There is evidence that 100 to 200 men per 1000 men screened will have a false-positive test. Most of these men will undergo prostate biopsy. A large proportion of patients with prostatic carcinomas detected by screening may be subjected to surgery, radiotherapy or androgen deprivation therapy. This article highlights the evidence against systematic screening. It proposes a holistic approach involving the individual in the discussion of the advantages and disadvantages of prostate cancer screening.

Keywords: Early Detection of Cancer; Mass Screening; Prostatic Neoplasms. 